\title{
The effect of transcutaneous spinal direct current stimulation on corticospinal excitability in chronic incomplete spinal cord injury
}

\author{
Elizabeth Salmon Powell ${ }^{\mathrm{a}}$, Cheryl Carrico ${ }^{\mathrm{a}}$, Emily Salyers ${ }^{\mathrm{a}}$, Philip M. Westgate \\ and Lumy Sawaki ${ }^{\mathrm{a}, \mathrm{c}, *}$ \\ ${ }^{a}$ Department of Physical Medicine and Rehabilitation, University of Kentucky, Lexington, KY, USA \\ ${ }^{\mathrm{b}}$ Department of Biostatistics, College of Public Health, University of Kentucky, Lexington, KY, USA \\ ${ }^{\mathrm{c}}$ HealthSouth Cardinal Hill Hospital, Lexington, KY, USA
}

\begin{abstract}
.
OBJECTIVES: This study investigated the feasibility of modulating bilateral corticospinal excitability with different polarities of transcutaneous spinal direct current stimulation (tsDCS) in chronic, incomplete spinal cord injury (SCI).

METHODS: Six subjects with chronic incomplete SCI (>12 months post injury) participated in this crossover study. Intervention consisted of 3 sessions, separated by at least 1 week, in which each subject received the conditions cathodal, anodal, and sham tsDCS. Stimulation was delivered at $2.5 \mathrm{~mA}$ for 20 minutes with the active electrode positioned over the spinous processes of T10-T11 and the reference electrode over left deltoid. To measure the effects of tsDCS on corticospinal excitability, motor evoked potentials (MEPs) from transcranial magnetic stimulation were measured bilaterally from soleus before and after tsDCS.

RESULTS: Five subjects completed all 3 sessions. One subject withdrew after 2 sessions due to complications unrelated to the study. MEPs were measurable in 5 subjects. No significant differences in change of MEP amplitudes were found between the 3 conditions. However, there were trends that indicated laterality of response, particularly with cathodal tsDCS increasing corticospinal excitability contralateral to the reference electrode and decreasing corticospinal excitability ipsilateral to the reference electrode.

CONCLUSION: Corticospinal excitability may be modulated with laterality by tsDCS in individuals with chronic, incomplete SCI. Further research is needed to 1) determine whether different placement of the reference electrode can lead to uniform modulation bilaterally, and 2) reveal whether these alterations in corticospinal excitability can lead to improved movement function in individuals with chronic, incomplete SCI.
\end{abstract}

Keywords: Neuromodulation, neuroplasticity, lower extremity, humans, transcranial magnetic stimulation

\footnotetext{
*Address for correspondence: Lumy Sawaki, University of Kentucky Department of Physical Medicine and Rehabilitation at Cardinal Hill Hospital, 2050 Versailles Road, Lexington, KY 40504, USA. Tel.: +1 859323 6226; Fax: +1 859323 1123; E-mail: 1sawa2@uky.edu.
}

\section{Introduction}

Approximately 12,000 new spinal cord injuries (SCIs) occur every year in the United States, and almost half of newly injured individuals are between 16 and 30 years of age ("Spinal cord injury facts and 
figures at a glance," 2014). It is estimated that less than $1 \%$ of individuals completely recover neurological function by the time of hospital discharge ("Spinal cord injury facts and figures at a glance," 2014). SCI often impairs the ability to walk; and diminished ability to walk is often associated with lower quality of life (Riggins, Kankipati, Oyster, Cooper, \& Boninger, 2011) and higher risk of developing comorbidities such as type 2 diabetes(Cragg, Noonan, Dvorak, et al., 2013) and cardiovascular disease (Cragg, Noonan, Krassioukov, \& Borisoff, 2013). No one form of locomotor training has been found to be superior to the others thus far (Mehrholz, Kugler, \& Pohl, 2012). Therefore, further research is needed to establish interventions to enhance long-term recovery of function and quality of life after SCI.

Recovery of function after SCI is due in part to neuroplastic change, or reorganization of the central nervous system (Behrman, Bowden, \& Nair, 2006; Dietz, 2011; Edgerton, Tillakaratne, Bigbee, de Leon, $\&$ Roy, 2004). Various forms of neuromodulation have been shown to drive neuroplastic change in healthy subjects (i.e., no neurological impairment) as well as subjects with SCI or other neurological impairments (Angeli, Edgerton, Gerasimenko, \& Harkema, 2014; Fregni \& Pascual-Leone, 2007; Priori, Ciocca, Parazzini, Vergari, \& Ferrucci, 2014). A noninvasive neuromodulation technique called transcranial direct current stimulation (tDCS) delivers electrical current through electrodes placed on the scalp (Nitsche, Liebetanz, Tergau, \& Paulus, 2002). The effects of tDCS differ based on the polarity of stimulation (Nitsche \& Paulus, 2000). The exact mechanisms of tDCS are not yet known, though it is theorized to affect N-methyl-D-aspartate (NMDA) receptors and gamma-aminobutyric acid (GABA) ergic activity (Brunoni et al., 2012). Several prior studies have investigated the use of tDCS in subjects with stroke (Edwards et al., 2009; Hummel et al., 2006; Kang, Summers, \& Cauraugh, 2016; Lee \& Lee, 2015). In such subjects, tDCS has been shown to improve movement function, such as increasing pinch force (Hummel et al., 2006). tDCS has also been shown to enhance outcomes of motor therapy measured with Fugl-Meyer Assessment (FMA) upper extremity motor scores (Lee $\&$ Lee, 2015) and increase motor evoked potential (MEP) amplitude measured with transcranial magnetic stimulation (TMS) (Edwards et al., 2009). While tDCS has not been studied extensively in subjects with SCI, active tDCS combined with locomotor training after SCI has been shown to improve scores on Manual Muscle Test, 6 Minute Walk Test, and Timed Up and Go more than sham tDCS (Raithatha et al., 2016).

Similar to tDCS, transcutaneous spinal direct current stimulation (tsDCS) is a noninvasive form of neuromodulation. The active electrode that determines stimulation polarity in tsDCS is generally placed over the thoracic spine; and the reference electrode is placed on either shoulder (Grecco, 2015; Priori et al., 2014). Animal studies indicate that similar to tDCS, tsDCS may affect GABAergic processes as well as glutamatergic and glycinergic processes (Priori et al., 2014). The effects of tsDCS are still under investigation, with the majority of studies to date having been conducted in healthy subjects without neurological impairment. In this population, anodal tsDCS has been found to decrease homosynaptic depression of soleus $\mathrm{H}$ reflex (Winkler, Hering, \& Straube, 2010) and induce a leftward shift of the soleus H-reflex recruitment curve (Lamy, Ho, Badel, Arrigo, \& Boakye, 2012). While it is still a matter of debate, these results suggest that tsDCS may modulate spinal reflexes and interneuronal spinal networks. Anodal tsDCS may lead to a decrease in the excitability of the entire corticospinal tract, as evidenced by the increase in resting motor threshold (rMT) in abductor hallucis (Bocci, Marceglia, et al., 2015). Conversely, cathodal tsDCS has been reported to affect spinal reflex activity through an increase in homosynaptic depression of soleus H-reflex (Winkler et al., 2010) and a decrease in the amplitude of the early component of the spinal reflex (Hubli, Dietz, SchraflAltermatt, \& Bolliger, 2013). Cathodal tsDCS has also been found to increase corticospinal excitability (i.e., increase MEP areas in upper and lower extremities) in healthy subjects without neurological impairment (Bocci, Marceglia, et al., 2015). However, Bocci et al. reported that only cathodal tsDCS was able to modulate spinal reflexes (Bocci et al., 2014). Both anodal and cathodal tsDCS have been reported to modulate transcallosal processing (Bocci, Caleo, et al., 2015; Schweizer, Meyer-Friessem, Zahn, Tegenthoff, \& Schmidt-Wilcke, 2017). In sum: for healthy individuals without neurological impairment, anodal tsDCS appears to decrease corticospinal excitability and increase spinal reflexes, while cathodal tsDCS appears to increase corticospinal excitability and decrease spinal reflexes; and both polarities may affect transcallosal processing. Therefore, it is conceivable that tsDCS may modulate spinal reflexes and interneuronal spinal networks, 
as well as corticospinal excitability and transcallosal processing, in subjects with SCI.

The ability to modulate reflexes could be beneficial in SCI, as the exaggerated activity of reflexes such as the spinal reflex and H-reflex are related to spasticity, which can reduce quality of life (Adams \& Hicks, 2005). A single study has investigated the use of tsDCS in subjects with complete SCI. Results showed that anodal tsDCS increased spinal reflex amplitude and decreased the threshold for eliciting the reflex ipsilateral to the reference electrode (Hubli et al., 2013). However, the study did not provide insight into how tsDCS may affect bilateral supraspinal excitability in SCI. To address this evidence gap, the present study evaluated the bilateral effects of differing polarities of tsDCS on supraspinal excitability in a small sample of subjects with motor incomplete SCI. As this would be only the second known study of tsDCS in SCI, a feasibility study was chosen due to two aspects of concern regarding SCI, the first of which is the presence of scar tissue. It is possible that the presence of scar tissue in the spinal cord could impede the flow of current through the cord. Also, SCIs can be heterogeneous with regard to lesion site. Therefore, another concern was whether heterogeneity of the lesion site, combined with the location of the reference electrode, would affect the results. The central hypothesis of this feasibility study was that cathodal tsDCS would result in increased MEPs representative of increased corticospinal excitability; that anodal tsDCS would result in decreased MEPs; and that sham tsDCS would not induce any notable changes in MEPs.

\section{Methods}

\subsection{Trial design}

The study used a single-blind, sham-controlled, randomized crossover design. Intervention consisted of 3 sessions, separated by at least 1 week. In each session, each subject received 1 of 3 conditions: cathodal tsDCS, anodal tsDCS, or sham tsDCS. Each subject received each condition once. All procedures were in accordance with the Declaration of Helsinki and were approved by the university Institutional Review Board. Written informed consent was obtained from all subjects prior to their enrollment in the study. All aspects of the study were performed in a university research clinic located within a rehabilitation hospital in the United States.

\subsection{Subjects}

Eligible subjects were between 18 and 65 years of age, with motor incomplete SCI at or above lumbar level Lland classified as C or D on the American Spinal Injury Association Impairment Scale. The exclusion criteria were: a) unstable cardiopulmonary conditions; b) history of seizure, head injury with loss of consciousness, severe alcohol or drug abuse, and/or psychiatric illness; c) cognitive deficits severe enough to preclude informed consent; d) positive pregnancy test or being of childbearing age and not using appropriate contraception; e) ferromagnetic material in the brain or in the spine (except for titanium for segmental fixation of the spine); f) cardiac or neural pacemakers; g) fixed contractures in the lower extremities; h) uncontrolled diabetes; i) severe osteoporosis; and/or j) severe spasticity.

\subsection{Intervention}

A NeuroConn Eldith direct current stimulator (Magstim, Whitland, Dyfed, UK) was used to deliver tsDCS through $4.5 \times 10 \mathrm{~cm}$ carbon electrodes (Covidien, Minneapolis, Minnesota) coated with EC2 conductive paste (Natus Neurology, Galway, Ireland). Skin areas under the electrodes were first prepped using an abrasive gel (NuPrep, Weaver and Company, Aurora, Colorado). Each of the following 3 conditions ( 1 in each session) was randomly administered to each subject: anodal tsDCS, cathodal tsDCS, and sham tsDCS.

For anodal and cathodal tsDCS, the active electrode (anode or cathode) was placed with its top edge aligned with the spinous process of T10. The reference electrode was placed horizontally over the left deltoid for all conditions. Electrode placement and stimulation was performed with the subject lying prone on a therapy plinth. tsDCS was delivered at $2.5 \mathrm{~mA}$ for a period of 20 minutes, resulting in a current density of $0.06 \mathrm{~mA} / \mathrm{cm}^{2}$ and a charge density of $0.07 \mathrm{C} / \mathrm{cm}^{2}$, which are well below the established safety limits for tDCS (Bikson, Datta, \& Elwassif, 2009). Stimulation current was ramped up over a period of 30 seconds at the onset of stimulation and back down over 30 seconds at stimulation offset.

For sham tsDCS, electrode placement was identical to active tsDCS. As with active tsDCS, current intensity was ramped up over 30 seconds at the onset of stimulation and back down over 30 seconds at stimulation offset; however, full-intensity stimulation only lasted 30 seconds; $0 \mathrm{~mA}$ was delivered 
for the remaining 8.5 minutes, and electrodes were left in place. Other studies using tDCS have shown that this protocol prevents subjects from differentiating between active and sham conditions (Gandiga, Hummel, \& Cohen, 2006). Because subjects had impaired sensation due to SCI, tsDCS was administered in 2 contiguous segments of 10 minutes' duration. Between segments, the skin was checked under both electrodes for signs of skin irritation. This checking was done irrespective of tsDCS condition.

\subsection{Outcome measures}

Corticospinal excitability was assessed via change in TMS MEP amplitudes in the right soleus (ie, MEP-RSol) and left soleus (ie, MEP-LSol) - the primary outcome measures - before and after tsDCS in each session. Disposable, pre-gelled recording electrodes (Lead-Lok, Inc., Sandpoint, Idaho) were placed halfway between the medial malleolus and the midline of the popliteal fossa. The reference electrodes were placed over the Achilles tendons; and the ground electrodes on the lateral portion of the leg, midway between the recording electrode and the popliteal fossa.

MEP amplitude data was collected with subjects in a seated position. Due to the time needed for subjects to transfer from prone to seated positions in between tsDCS and TMS, an approximate average of 20 minutes elapsed between the offset of tsDCS and the collection of post-tsDCS MEPs. TMS was delivered with a Magstim $200^{2}$ stimulator connected to a double-cone coil. Coil positioning was guided using the Brainsight ${ }^{\mathrm{TM}}$ frameless stereotaxy system (Rogue Research Inc., Montreal, Quebec, Canada), which indicates the coil position on a curvilinear reconstruction of a template MRI. The TMS hot-spot (ie, the stimulation location that elicits the largest MEP in the target muscle) was localized bilaterally. The resting motor threshold, or rMT (ie, the lowest stimulation intensity that results in at least 5 of 10 consecutive MEPs $\geq 50 \mu \mathrm{V}$ ), was also determined using the Rossini-Rothwell method (Tranulis et al., 2006). Five stimuli, with an interstimulus interval of 5 seconds, were delivered to the hotspot at $110 \%$ rMT. The resulting MEP amplitudes were analyzed.

\subsection{Statistics}

Changes in MEP-RSol and MEP-LSol values from pre to post were the outcomes of interest. Because subjects had change scores measured for each treatment condition, repeated measures analyses were conducted using a multivariate Gaussian linear model incorporating a working unstructured covariance structure. All tests were 2 -sided with statistical significance pre-specified as $P<0.05$. Analyses were conducted in SAS version 9.4 (SAS Institute, Cary, $\mathrm{NC})$.

\section{Results}

Subject demographics are shown in Table 1. One subject withdrew from the study after 2 sessions due to health issues unrelated to the study. Available data from the 2 sessions was included in analysis. All other subjects completed all 3 sessions per protocol. MEPs were not elicited from some subjects, even

Table 1

Subject demographics and clinical characteristics

\begin{tabular}{|c|c|c|c|c|c|c|c|c|c|}
\hline \multirow{2}{*}{$\begin{array}{l}\text { Subject } \\
\text { number }\end{array}$} & \multirow{2}{*}{$\begin{array}{l}\text { Age } \\
\text { (years) }\end{array}$} & \multirow[t]{2}{*}{ Sex } & \multirow{2}{*}{$\begin{array}{l}\text { Time } \\
\text { since SCI }\end{array}$} & \multirow{2}{*}{$\begin{array}{l}\text { AIS } \\
\text { Score }\end{array}$} & \multirow{2}{*}{$\begin{array}{l}\text { Level } \\
\text { of SCI }\end{array}$} & \multirow{2}{*}{$\begin{array}{l}\text { Measurable } \\
\text { MEPs }\end{array}$} & \multicolumn{3}{|c|}{ Condition } \\
\hline & & & & & & & Session 1 & Session 2 & Session 3 \\
\hline 1 & 31 & M & 164 & $\mathrm{C}$ & C6 & $\begin{array}{l}\text { Yes, only } \\
\text { during } \\
\text { anodal } \\
\text { session }\end{array}$ & Cathodal & Anodal & Sham \\
\hline 2 & 55 & M & 175 & $\mathrm{C}$ & $\mathrm{T} 2$ & Yes & Anodal & Sham & N/A \\
\hline 3 & 48 & $\mathrm{~F}$ & 186 & $\mathrm{C}$ & T6 & No & Anodal & Sham & Cathodal \\
\hline 4 & 59 & M & 58 & $\mathrm{D}$ & L1 & Yes & Anodal & Sham & Cathodal \\
\hline 5 & 56 & $\mathrm{~F}$ & 493 & $\mathrm{D}$ & $\mathrm{L} 1$ & Yes & Anodal & Cathodal & Sham \\
\hline 6 & 26 & M & 55 & $\mathrm{C}$ & T6 & $\begin{array}{l}\text { Yes, only } \\
\text { on right } \\
\text { leg }\end{array}$ & Sham & Cathodal & Anodal \\
\hline
\end{tabular}

SCI: spinal cord injury. AIS: American Spinal Injury Association Impairment Scale. Time since SCI: in months. MEPs: motor evoked potentials. M: male. F: female. 
with stimulator output at $100 \%$. These subjects are indicated in Table 1. In these cases, pre and post MEP values were considered to be $0 \mu \mathrm{V}$ and included in analysis. Pre and post results for individuals are shown in Table 2. Representative MEP tracings from subject 5 from each condition are shown in Fig. 1. Results of group-wise analysis are shown in Table 3. Within-group analysis revealed no significant prepost changes in bilateral MEP amplitude for anodal tsDCS, cathodal tsDCS, or sham tsDCS. Betweengroup analysis revealed no significant differences (Fig. 2).

\section{Discussion}

This feasibility study was the first to use a crossover design to evaluate the supraspinal effects of tsDCS on bilateral MEPs in people with SCI. While larger studies will be required to establish the supraspinal effects of tsDCS after SCI, cathodal tsDCS induced an increase in MEP-RSol, contralateral to the reference electrode, and a decrease in MEP-LSol, ipsilateral to the reference electrode. Further, anodal tsDCS induced an increase in MEP-LSol. Although the magnitude of these changes did not reach significance, they indicate that cathodal and anodal tsDCS may be capable of modulating corticospinal excitability in people with SCI.

Cathodal tsDCS led to similar results as a previous study with a larger sample size in healthy subjects without SCI (Bocci, Marceglia, et al., 2015).
Specifically, Bocci and colleagues investigated the effects of cathodal and anodal tsDCS on MEP areas of the abductor hallucis contralateral to the reference tsDCS electrode (Bocci, Marceglia, et al., 2015). Results showed an increased MEP area associated with cathodal tsDCS and decreased MEP area associated with anodal tsDCS. Direction of change following cathodal tsDCS (Bocci, Marceglia, et al., 2015) was consistent with the direction of change in MEP-RSol amplitude in the present study. No other studies have measured the bilateral effects of tsDCS on MEP amplitude.

In the present study, the direction of MEP-LSol change differed from the direction of MEP-RSol change following cathodal tsDCS. Moreover, while cathodal tsDCS induced a greater increase in MEPRSol than anodal or sham tsDCS, it also induced a slight decrease in MEP-LSol. These effects may be indicative that cathodal tsDCS modulates transcallosal processing (TCP). TCP is a normally occurring process in which one brain hemisphere provides inhibitory inputs to the other hemisphere (Davidson \& Tremblay, 2013; Perez \& Cohen, 2009). tDCS (i.e., brain stimulation) has been shown to modulate the duration of TCP dependent on polarity of stimulation (Lang, Nitsche, Paulus, Rothwell, \& Lemon, 2004), and tsDCS has been shown to have supraspinal effects (Bocci, Caleo, et al., 2015; Bocci, Marceglia, et al., 2015). Therefore, it is conceivable that cathodal tsDCS may increase TCP (i.e., left brain inhibition of right brain) along with increasing excitability of the left brain and spinal projections to the right leg.

Table 2

Resting motor thresholds (\% maximum stimulator output), motor evoked potential means (standard errors) by subject

\begin{tabular}{|c|c|c|c|c|c|c|c|c|c|c|c|c|}
\hline \multirow{3}{*}{$\begin{array}{l}\text { Subject } \\
\text { number }\end{array}$} & \multicolumn{4}{|c|}{ Anodal } & \multicolumn{4}{|c|}{ Cathodal } & \multicolumn{4}{|c|}{ Sham } \\
\hline & \multicolumn{2}{|c|}{ Right Leg } & \multicolumn{2}{|c|}{ Left Leg } & \multicolumn{2}{|c|}{ Right Leg } & \multicolumn{2}{|c|}{ Left Leg } & \multicolumn{2}{|c|}{ Right Leg } & \multicolumn{2}{|c|}{ Left Leg } \\
\hline & Pre & Post & Pre & Post & Pre & Post & $\overline{\text { Pre }}$ & Post & $\overline{P r e}$ & Post & Pre & Post \\
\hline 1 & - & - & $\begin{array}{c}85 \% \\
29 \\
(14)\end{array}$ & $\begin{array}{c}87 \% \\
77 \\
(15)\end{array}$ & - & - & - & - & - & - & - & - \\
\hline 2 & $\begin{array}{c}61 \% \\
48 \\
(3)\end{array}$ & $\begin{array}{c}46 \% \\
56 \\
(2)\end{array}$ & $\begin{array}{r}60 \% \\
140 \\
(14)\end{array}$ & $\begin{array}{c}54 \% \\
158 \\
(52)\end{array}$ & - & - & - & - & $\begin{array}{c}72 \% \\
56 \\
(2)\end{array}$ & $\begin{array}{c}66 \% \\
62 \\
(2)\end{array}$ & $\begin{array}{c}58 \% \\
126 \\
(59)\end{array}$ & $\begin{array}{c}55 \% \\
99 \\
(8)\end{array}$ \\
\hline 3 & - & - & - & - & - & - & - & - & - & - & - & - \\
\hline 4 & $\begin{array}{c}62 \% \\
88 \\
(11)\end{array}$ & N/A* & $\begin{array}{c}42 \% \\
131 \\
(26)\end{array}$ & $\begin{array}{c}49 \% \\
202 \\
(49)\end{array}$ & $\begin{array}{c}50 \% \\
80 \\
(15)\end{array}$ & $\begin{array}{c}50 \% \\
139 \\
(38)\end{array}$ & $\begin{array}{c}52 \% \\
136 \\
(34)\end{array}$ & $\begin{array}{c}41 \% \\
113 \\
(28)\end{array}$ & $\begin{array}{c}53 \% \\
88 \\
(24)\end{array}$ & $\begin{array}{c}58 \% \\
140 \\
(42)\end{array}$ & $\begin{array}{c}43 \% \\
66 \\
(11)\end{array}$ & $\begin{array}{c}46 \% \\
147 \\
(41)\end{array}$ \\
\hline 5 & $\begin{array}{c}70 \% \\
61 \\
(16)\end{array}$ & $\begin{array}{c}78 \% \\
75 \\
(16)\end{array}$ & $\begin{array}{c}82 \% \\
25 \\
(2)\end{array}$ & $\begin{array}{c}81 \% \\
36 \\
(9)\end{array}$ & $\begin{array}{c}71 \% \\
57 \\
(15)\end{array}$ & $\begin{array}{c}81 \% \\
85 \\
(7)\end{array}$ & $\begin{array}{c}62 \% \\
37 \\
(5)\end{array}$ & $\begin{array}{c}68 \% \\
26 \\
(4)\end{array}$ & $\begin{array}{c}74 \% \\
67 \\
(7)\end{array}$ & $\begin{array}{c}62 \% \\
48 \\
(14)\end{array}$ & $\begin{array}{c}69 \% \\
32 \\
(5)\end{array}$ & $\begin{array}{c}72 \% \\
28 \\
(7)\end{array}$ \\
\hline 6 & $\begin{array}{c}75 \% \\
61 \\
(9)\end{array}$ & $\begin{array}{c}78 \% \\
41 \\
(3)\end{array}$ & - & - & $\begin{array}{c}76 \% \\
36 \\
(7)\end{array}$ & $\begin{array}{c}76 \% \\
76 \\
(6)\end{array}$ & - & - & $\begin{array}{c}72 \% \\
55 \\
(18)\end{array}$ & $\begin{array}{c}78 \% \\
32 \\
(6)\end{array}$ & - & - \\
\hline
\end{tabular}

-: motor evoked potentials could not be produced in these cases. N/A*: post data could not be collected due to time constraints. 

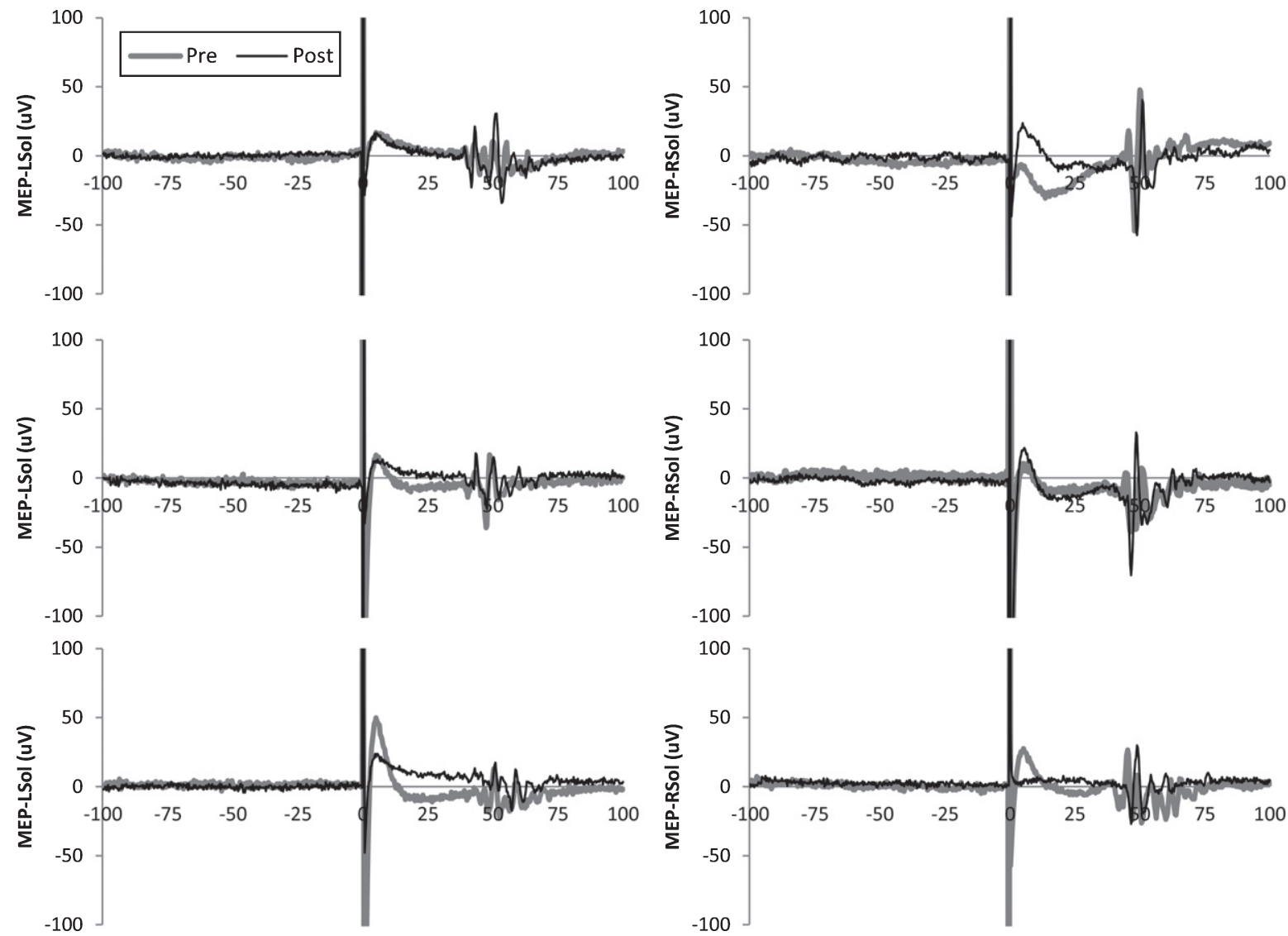

Fig. 1. Representative motor evoked potentials (MEPs) from subject 5. Single MEPs from before and after transcutaneous spinal direct current stimulation (tsDCS) are shown for each leg and condition.

Table 3

Estimated means, standard errors, and $p$-values mean change in motor evoked potentials from pre- to post-tsDCS

\begin{tabular}{lcc}
\hline & Right Leg/Left Brain & Left Leg/Right Brain \\
\hline Anodal & $4.23(8.30)$ & $24.67(11.75)$ \\
& $P=0.70$ & $p=0.09$ \\
Cathodal & $25.66(12.04)$ & $-5.06(4.44)$ \\
Sham & $p=0.09$ & $p=0.31$ \\
& $2.67(10.94)$ & $8.33(15.15)$ \\
Anodal - Cathodal & $p=0.82$ & $p=0.61$ \\
& $-21.43(13.35)$ & $29.72(14.84)$ \\
Anodal - Sham & $p=0.19$ & $p=0.10$ \\
& $1.56(9.79)$ & $16.33(10.09)$ \\
Cathodal - Sham & $p=0.90$ & $p=0.17$ \\
& $22.99(13.17)$ & $-13.39(18.98)$ \\
& $p=0.14$ & $p=0.51$ \\
\hline
\end{tabular}

This dynamic would explain the small decrease in MEP-LSol observed in the present study. The lack of a decrease in MEP-RSol, contralateral to the reference, following anodal tsDCS indicates a lack of TCP modulation associated with this polarity, suggesting different mechanisms of action of anodal and cathodal tsDCS. This indicates that cathodal tsDCS may have increased activity of excitatory transcallosal fibers of the left brain which act on inhibitory interneurons of the right brain (Lang et al., 2004). Future studies are needed to substantiate whether cathodal tsDCS specifically modulates TCP.

Differential effects of tsDCS on MEP-RSol and MEP-LSol have not been investigated in previous studies. Results of the present study suggest that in cases of motor incomplete SCI, cathodal tsDCS may induce the greatest change in MEP amplitude in the lower extremity contralateral to the reference electrode, while anodal tsDCS will induce the greatest change in the lower extremity ipsilateral to the reference electrode. However, further studies are needed to evaluate the relationship between laterality of effects and the right and left neurological levels of subjects.

Limitations of the present study include small sample size as well as sample heterogeneity with regards to time since injury and level and completeness of injury. The chronicity of the injury may influence the 

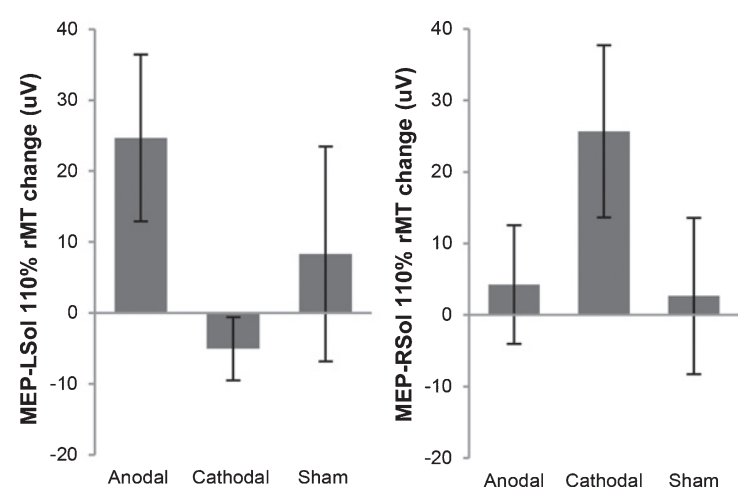

Fig. 2. Motor evoked potential (MEP) change in amplitude from before tsDCS to after tsDCS. MEPs were measured at $110 \%$ of resting motor threshold (rMT). MEPs resulting from stimulation of the right motor cortex, measured from the left soleus (MEPLSol), ipsilateral to the reference electrode, increased following anodal and sham tsDCS, and decreased following cathodal tsDCS. None of these changes were significant. There were no significant differences between the 3 groups. MEPs resulting from stimulation of the left motor cortex, measured from the right soleus (MEP$\mathrm{RSol}$ ), contralateral to the reference electrode, increased following anodal, cathodal, and sham tsDCS. None of these changes were significant. There were no significant differences between the 3 conditions. Error bars indicate standard error.

effects of tsDCS, as may the level and completeness of the injury. Heterogeneity of these factors likely increased the variance of the results. Furthermore, upper extremity MEPs were not measured in this study in an attempt to limit the time required of the subjects. However, measurement of upper extremity MEPs in addition to lower extremity MEPs would give a more complete assessment of the effects of tsDCS. The small sample size is an issue which was exacerbated by the lack of measurable MEPs in multiple subjects. Because individuals with SCI have fewer intact fibers in the corticospinal tract, MEP amplitudes or other indicators of corticospinal excitability are decreased compared to neurologically intact individuals (Roy, Yang, \& Gorassini, 2010). There are several methods for handling non-recordable MEPs. One such method is to analyze the maximum value for motor threshold; however, this method would inflate the sample (Butefisch et al., 2002). Another method is to use multimodal evoked potential scores (Jung, Beyerle, \& Ziemann, 2008). However, this method was developed for use in multiple sclerosis populations and would require modifications and validation before application in traumatic SCI. By simply excluding these subjects from the data analysis, the data would then be skewed to represent only the subjects with responses (Butefisch,
Wessling, Netz, Seitz, \& Homberg, 2008; Schambra et al., 2015; Swayne, Rothwell, Ward, \& Greenwood, 2008). Therefore, in order to decrease biases, amplitudes of $0 \mu \mathrm{V}$ were used in instances where MEPs could not be measured, as was done in previous studies (Sawaki et al., 2014; Traversa et al., 2000). Future studies could screen all subjects prior to enrollment to ensure that MEPs can be elicited from the soleus or other target muscles. Alternatively, enrollment could be limited to individuals with American Spinal Injury Association Impairment Scale (AIS) scores of D, as lower extremity MEPs were easier to obtain from subjects with AIS scores of D in this study than from those with AIS scores of C. The inclusion of AIS D subjects will likely be integral to determining the mechanisms of action of tsDCS in the SCI population. Additionally, TMS should be administered with subjects in a standing frame. In healthy subjects with no neurological impairment, standing has been shown to increase corticospinal excitability compared with sitting, as measured with maximum MEP size and recruitment curve slopes of soleus and tibialis anterior (TA) (Obata, Sekiguchi, Nakazawa, \& Ohtsuki, 2009). Also, MEPs should be measured from TA rather than soleus while subjects are in a standing frame. Soleus was chosen for the present study because it is a commonly used muscle in measurement of H-reflex (Knikou, 2008; Little \& Halar, 1985; Nakazawa, Kawashima, \& Akai, 2006), a measure of spinal excitability that is targeted in tsDCS and is altered in individuals with SCI (Knikou, Angeli, Ferreira, \& Harkema, 2009; Sayenko, Masani, Alizadeh-Meghrazi, Popovic, \& Craven, 2010). At the same time, several studies in healthy subjects with no neurological impairment have indicated that TA may be the optimal target for eliciting MEPs. For example, Ackermann and colleagues (1991) determined rMT of TA with subjects in supine. Stimuli were then delivered at rMT, as well as at $10 \%$ above rMT, with subjects in supine as well as during standing. MEP amplitudes in TA were significantly larger while standing compared to supine. Similarly, when rMT was measured while standing, TA MEP areas were found to be significantly larger at 5\% and $10 \%$ above rMT while standing as compared to supine (Nakazawa et al., 2003). Soleus MEP areas did not vary significantly between supine and standing. In a separate study, Obata and colleagues (2009) determined that the rMTs of soleus and TA do not vary between standing and sitting. However, maximum slopes of the recruitment curves were significantly greater in 
both the soleus and TA when standing as compared to sitting. The increase in the maximum TA slope was significantly greater than the increase in soleus slope. Given this evidence, it appears that measuring MEPs from TA in subjects with SCI may be more optimal than measuring MEPs from soleus in terms of yielding responses.

These preliminary results indicate the ability of cathodal tsDCS to modulate corticospinal excitability with marked laterality of effects. This evidence warrants a larger study. Further studies are needed for systematically evaluating the impact of the reference electrode placement on cortical and spinal excitability. Additional studies with larger sample sizes are needed to enable better understanding of the effects of tsDCS on corticospinal excitability and to determine whether these effects can support functional improvements for people with chronic, motor incomplete SCI.

\section{Acknowledgments}

We extend our heartfelt thanks to our study participants.

\section{Conflict of interest}

This publication was supported by the National Center for Research Resources and the National Center for Advancing Translational Sciences, National Institutes of Health, through Grant UL1TR000117, and the HealthSouth Cardinal Hill Stroke and Spinal Cord Endowment (1215375670).

\section{References}

Ackermann, H., Scholz, E., Koehler, W., \& Dichgans, J. (1991). Influence of posture and voluntary background contraction upon compound muscle action potentials from anterior tibial and soleus muscle following transcranial magnetic stimulation. Electroencephalogr Clin Neurophysiol, 81(1), 71-80.

Adams, M. M., \& Hicks, A. L. (2005). Spasticity after spinal cord injury. Spinal Cord, 43(10), 577-586. doi:10.1038/sj.sc.3101757

Angeli, C. A., Edgerton, V. R., Gerasimenko, Y. P., \& Harkema, S. J. (2014). Altering spinal cord excitability enables voluntary movements after chronic complete paralysis in humans. Brain, 137(Pt 5), 1394-1409. doi:10.1093/brain/awu038

Behrman, A. L., Bowden, M. G., \& Nair, P. M. (2006). Neuroplasticity after spinal cord injury and training: An emerging paradigm shift in rehabilitation and walking recovery. Phys Ther, 86(10), 1406-1425. doi:10.2522/ptj.20050212

Bikson, M., Datta, A., \& Elwassif, M. (2009). Establishing safety limits for transcranial direct current stimulation. Clin Neurophysiol, 120(6), 1033-1034.

Bocci, T., Caleo, M., Vannini, B., Vergari, M., Cogiamanian, F., Rossi, S., ...\& Sartucci, F. (2015). An unexpected target of spinal direct current stimulation: Interhemispheric connectivity in humans. Journal of Neuroscience Methods, 254, 18-26. doi:10.1016/j.jneumeth.2015.07.012

Bocci, T., Marceglia, S., Vergari, M., Cognetto, V., Cogiamanian, F., Sartucci, F., \& Priori, A. (2015). Transcutaneous spinal direct current stimulation modulates human corticospinal system excitability. Journal of Neurophysiology, 114(1), 440-446. doi:10.1152/jn.00490.2014

Bocci, T., Vannini, B., Torzini, A., Mazzatenta, A., Vergari, M., Cogiamanian, F., ...\& \& Sartucci, F. (2014). Cathodal transcutaneous spinal direct current stimulation (tsDCS) improves motor unit recruitment in healthy subjects. Neuroscience Letters, 578, 75-79. doi:10.1016/j.neulet.2014.06.037

Brunoni, A. R., Nitsche, M. A., Bolognini, N., Bikson, M., Wagner, T., Merabet, L., ...\& Pascual-Leone, A. (2012). Clinical research with transcranial direct current stimulation (tDCS): Challenges and future directions. Brain Stimul, 5(3), 175-195.

Butefisch, C. M., Davis, B. C., Sawaki, L., Waldvogel, D., Classen, J., Kopylev, L., \& Cohen, L. G. (2002). Modulation of usedependent plasticity by d-amphetamine. Annals of Neurology, 51(1), 59-68.

Butefisch, C. M., Wessling, M., Netz, J., Seitz, R. J., \& Homberg, V. (2008). Relationship between interhemispheric inhibition and motor cortex excitability in subacute stroke patients. Neurorehabil Neural Repair, 22(1), 4-21. doi:10.1177/1545968307301769

Cragg, J. J., Noonan, V. K., Dvorak, M., Krassioukov, A., Mancini, G. B., \& Borisoff, J. F. (2013). Spinal cord injury and type 2 diabetes: Results from a population health survey. Neurology, 81(21), 1864-1868. doi:10.1212/01.wnl.0000436074.98534.6e

Cragg, J. J., Noonan, V. K., Krassioukov, A., \& Borisoff, J. (2013). Cardiovascular disease and spinal cord injury: Results from a national population health survey. Neurology, 81(8), 723-728. doi:10.1212/WNL.0b013e3182a1aa68

Davidson, T., \& Tremblay, F. (2013). Age and hemispheric differences in transcallosal inhibition between motor cortices: An ipsilateral silent period study. BMC Neuroscience, 14, 62. doi:10.1186/1471-2202-14-62

Dietz, V. (2011). Neuronal plasticity after a human spinal cord injury: Positive and negative effects. Exp Neurol. doi:10.1016/j.expneurol.2011.04.007

Edgerton, V. R., Tillakaratne, N. J., Bigbee, A. J., de Leon, R. D., \& Roy, R. R. (2004). Plasticity of the spinal neural circuitry after injury. Annu Rev Neurosci, 27, 145-167. doi:10.1146/annurev.neuro.27.070203.144308

Edwards, D. J., Krebs, H. I., Rykman, A., Zipse, J., Thickbroom, G. W., Mastaglia, F. L., .. \&\& Volpe, B. T. (2009). Raised corticomotor excitability of M1 forearm area following anodal tDCS is sustained during robotic wrist therapy in chronic stroke. Restorative Neurology and Neuroscience, 27(3), 199207. doi:10.3233/rnn-2009-0470

Fregni, F., \& Pascual-Leone, A. (2007). Technology insight: Noninvasive brain stimulation in neurology-perspectives on the 
therapeutic potential of rTMS and tDCS. Nat Clin Pract Neurol, 3(7), 383-393. doi:10.1038/ncpneuro0530

Gandiga, P. C., Hummel, F. C., \& Cohen, L. G. (2006). Transcranial DC stimulation (tDCS): A tool for double-blind sham-controlled clinical studies in brain stimulation. Clin Neurophysiol, 117(4), 845-850. doi:10.1016/j.clinph.2005.12.003

Grecco, L. H. (2015). Transcutaneous spinal stimulation as a therapeutic strategy for spinal cord injury: State of the art. Journal of Neurorestoratology, 3, 73-82.

Hubli, M., Dietz, V., Schrafl-Altermatt, M., \& Bolliger, M. (2013). Modulation of spinal neuronal excitability by spinal direct currents and locomotion after spinal cord injury. Clin Neurophysiol, 124(6), 1187-1195. doi:10.1016/j.clinph.2012. 11.021

Hummel, F. C., Voller, B., Celnik, P., Floel, A., Giraux, P., Gerloff, C., \& Cohen, L. G. (2006). Effects of brain polarization on reaction times and pinch force in chronic stroke. BMC Neurosci, 7 , 73. doi:10.1186/1471-2202-7-73

Jung, P., Beyerle, A., \& Ziemann, U. (2008). Multimodal evoked potentials measure and predict disability progression in early relapsing-remitting multiple sclerosis. Multiple Sclerosis, 14(4), 553-556. doi:10.1177/1352458507085758

Kang, N., Summers, J. J., \& Cauraugh, J. H. (2016). Transcranial direct current stimulation facilitates motor learning post-stroke: A systematic review and meta-analysis. Journal of Neurology, Neurosurgery and Psychiatry, 87(4), 345-355. doi:10.1136/jnnp-2015-311242

Knikou, M. (2008). The H-reflex as a probe: Pathways and pitfalls. J Neurosci Methods, 171(1), 1-12. doi:10.1016/j.jneumeth.2008.02.012

Knikou, M., Angeli, C. A., Ferreira, C. K., \& Harkema, S. J. (2009). Soleus H-reflex gain, threshold, and amplitude as function of body posture and load in spinal cord intact and injured subjects. Int J Neurosci, 119(11), 2056-2073.

Lamy, J. C., Ho, C., Badel, A., Arrigo, R. T., \& Boakye, M. (2012). Modulation of soleus $\mathrm{H}$ reflex by spinal DC stimulation in humans. Journal of Neurophysiology, 108(3), 906-914. doi:10.1152/jn.10898.2011

Lang, N., Nitsche, M. A., Paulus, W., Rothwell, J. C., \& Lemon, R. N. (2004). Effects of transcranial direct current stimulation over the human motor cortex on corticospinal and transcallosal excitability. Experimental Brain Research, 156(4), 439-443. doi:10.1007/s00221-003-1800-2

Lee, D. G., \& Lee, D. Y. (2015). Effects of adjustment of transcranial direct current stimulation on motor function of the upper extremity in stroke patients. J Phys Ther Sci, 27(11), 3511-3513. doi:10.1589/jpts.27.3511

Little, J. W., \& Halar, E. M. (1985). H-reflex changes following spinal cord injury. Arch Phys Med Rehabil, 66(1), 19-22.

Mehrholz, J., Kugler, J., \& Pohl, M. (2012). Locomotor training for walking after spinal cord injury. Cochrane Database Syst Rev, 11, Cd006676. doi:10.1002/14651858.CD006676. pub3

Nakazawa, K., Kawashima, N., \& Akai, M. (2006). Enhanced stretch reflex excitability of the soleus muscle in persons with incomplete rather than complete chronic spinal cord injury. Archives of Physical Medicine and Rehabilitation, 87(1), 7175. doi:10.1016/j.apmr.2005.08.122

Nakazawa, K., Kawashima, N., Obata, H., Yamanaka, K., Nozaki, D., \& Akai, M. (2003). Facilitation of both stretch reflex and corticospinal pathways of the tibialis anterior muscle during standing in humans. Neuroscience Letters, 338(1), 53-56.

Nitsche, M. A., Liebetanz, D., Tergau, F., \& Paulus, W. (2002). Modulation of cortical excitability by transcranial direct current stimulation. Nervenarzt, 73(4), 332-335.

Nitsche, M. A., \& Paulus, W. (2000). Excitability changes induced in the human motor cortex by weak transcranial direct current stimulation. Journal of Physiology, 527(Pt 3), 633-639.

Obata, H., Sekiguchi, H., Nakazawa, K., \& Ohtsuki, T. (2009). Enhanced excitability of the corticospinal pathway of the ankle extensor and flexor muscles during standing in humans. Experimental Brain Research, 197(3), 207-213. doi:10.1007/s00221-009-1874-6

Perez, M. A., \& Cohen, L. G. (2009). Interhemispheric inhibition between primary motor cortices: What have we learned? Journal of Physiology, 587(Pt 4), 725-726. doi:10.1113/jphysiol.2008.166926

Priori, A., Ciocca, M., Parazzini, M., Vergari, M., \& Ferrucci, R. (2014). Transcranial cerebellar direct current stimulation and transcutaneous spinal cord direct current stimulation as innovative tools for neuroscientists. J Physiol London, 592(16), 3345-3369. doi:10.1113/jphysiol.2013.270280

Raithatha, R., Carrico, C., Powell, E. S., Westgate, P. M., Chelette Ii, K. C., Lee, K., .. \& \& Sawaki, L. (2016). Non-invasive brain stimulation and robot-assisted gait training after incomplete spinal cord injury: A randomized pilot study. NeuroRehabilitation, 38(1), 15-25. doi:10.3233/nre-151291

Riggins, M. S., Kankipati, P., Oyster, M. L., Cooper, R. A., \& Boninger, M. L. (2011). The relationship between quality of life and change in mobility 1 year postinjury in individuals with spinal cord injury. Archives of Physical Medicine and Rehabilitation, 92(7), 1027-1033. doi:10.1016/j.apmr.2011. 02.010

Roy, F. D., Yang, J. F., \& Gorassini, M. A. (2010). Afferent Regulation of Leg Motor Cortex Excitability After Incomplete Spinal Cord Injury. Journal of Neurophysiology, 103(4), 2222-2233. doi:10.1152/jn.00903.2009

Sawaki, L., Butler, A. J., Leng, X., Wassenaar, P. A., Mohammad, Y. M., Blanton, S., ...\& Wittenberg, G. F. (2014). Differential patterns of cortical reorganization following constraint-induced movement therapy during early and late period after stroke: A preliminary study. NeuroRehabilitation. doi:10.3233/NRE-141132

Sayenko, D. G., Masani, K., Alizadeh-Meghrazi, M., Popovic, M. R., \& Craven, B. C. (2010). Acute effects of whole body vibration during passive standing on soleus $\mathrm{H}$-reflex in subjects with and without spinal cord injury. Neurosci Lett, 482(1), 66-70. doi:10.1016/j.neulet.2010.07.009

Schambra, H. M., Ogden, R. T., Martinez-Hernandez, I. E., Lin, X., Chang, Y. B., Rahman, A., ...\& Krakauer, J. W. (2015). The reliability of repeated TMS measures in older adults and in patients with subacute and chronic stroke. Frontiers in Cellular Neuroscience, 9, 335. doi:10.3389/fncel.2015. 00335

Schweizer, L., Meyer-Friessem, C. H., Zahn, P. K., Tegenthoff, M., \& Schmidt-Wilcke, T. (2017). Transcutaneous Spinal Direct Current Stimulation Alters Resting-State Functional Connectivity. Brain Connect, 7(6), 357-365. doi:10.1089/brain.2017.0505 
Spinal cord injury facts and figures at a glance. (2014). $J$ Spinal Cord Med, 37(1), 117-118. doi:10.1179/1079026813Z. 000000000249

Swayne, O. B., Rothwell, J. C., Ward, N. S., \& Greenwood, R. J. (2008). Stages of motor output reorganization after hemispheric stroke suggested by longitudinal studies of cortical physiology. Cereb Cortex, 18(8), 1909-1922. doi:10.1093/cercor/bhm218

Tranulis, C., Gueguen, B., Pham-Scottez, A., Vacheron, M. N., Cabelguen, G., Costantini, A., ...\& Galinovski, A. (2006). Motor threshold in transcranial magnetic stimulation: Comparison of three estimation methods. Neurophysiologie Clinique, 36(1), 1-7. doi:10.1016/j.neucli.2006.01.005
Traversa, R., Cicinelli, P., Oliveri, M., Giuseppina Palmieri, M., Filippi, M. M., Pasqualetti, P., \& Rossini, P. M. (2000). Neurophysiological follow-up of motor cortical output in stroke patients. Clin Neurophysiol, 111(9), 1695-1703.

Winkler, T., Hering, P., \& Straube, A. (2010). Spinal DC stimulation in humans modulates post-activation depression of the H-reflex depending on current polarity. Clin Neurophysiol, 121(6), 957-961. doi:10.1016/j.clinph.2010.01.014 